\title{
PSEUDOTUMOR CEREBRAL SEM EDEMA DE PAPILA OPTICA
}

\author{
J. C. BRANT SEgGIA *, M. L. BEZERRA DE MENEZES * *
}

\begin{abstract}
RESUMO - Os autores apresentam quatro casos de síndrome de hipertensão intracraniana benigna em duas mulheres e dois homens jovens e magros em que todos os critérios diagnósticos foram preenchidos exceto a presença de edema de papila óptica. Considerando que casos semeIhantes já foram descritos por outros, pareceu-lhes justificada a proposição que fazem de não ser obrigatória a presença de edema de papila óptica para o diagnóstico da síndrome. Apresentam uma hipótese para explicar o fato e discutem outros detalhes dos casos.
\end{abstract}

PALAVRAS-CHAVE: pseudotumor cerebral, hipertensão intracraniana benigna, papilas ópticas normais.

Pseudotumor cerebri in the absence of papilledema.

SUMMARY - Four cases of benign intracranial hypertension in two nonobese women (23 and $35 \mathrm{yr}$. old) and two nonobese men (both $47 \mathrm{yr}$. old) are presented. The most remarkable finding of the clinical investigation of these cases was the absence of papilledema. Our finding is consistent with other reports, and suggest that the presence of papilledema should not be considered critical for the diagnosis of benign intracranial hypertension. Additionally, the authors present a hypothesis for the absence of papilledema in cases otherwise typical of benign intracranial hypertension.

KEY WORDS: pseudotumor cerebri, benign intracranial hypertension, normal optic papillac.

Pseudotumor cerebri ou síndrome de hipertensão intracraniana benigna é termo coletivo para diversos complexos sintomáticos caracterizados por aumento da pressão intracraniana, papiledema e alargamento da mancha cega; nenhum sinal focal exceto ocasional paralisia do sexto nervo craniano, composição do líquido cefalorraquidiano (LCR) normal e tomografia computadorizada (TC) sem evidências de massa intracraniana e alargamento ventricular ${ }^{43}$. Devemos excluir trombose de seios venosos intracranianos, infecção intracraniana, obstrução de ventrículos cerebrais, encefalopatia hipertensiva e retenção crônica de bióxido de carbono.

Apresentamos quatro casos de síndrome de pseudotumor cerebri que, apesar de preencherem todos os outros critérios diagnósticos, não apresentavam edema de papila óptica. Discutimos os achados clínicos e os estudamos comparativamente aos publicados na literatura. Acreditamos ser necessário reconsiderar o critério "presença de papiledema» para o diagnóstico.

\section{RELATO dOS CASOS}

Caso 1. VCA, 23 anos de idade, solteira, enfermeira, dextra. Natural e residente no Rio de Janeiro. Suas queixas iniciam-se 10 dias antes da consulta com cefaléia surda, intensa, holocraniana, contínua e de caracteritica nunca experimentada antes. Não era acompanhada de vômitos ou rigidez de nuca. Sob questionário informou que há três anos sofreu acidente automobilistico com traumatismo craniano leve e sem maiores consequências. Nega uso de anti-

* Chefe de Clínica do Serviço de Neurologia do Hospital dos Servidores do Estado/ INAMPS, Rio de Janeiro (HSE); ** Médico Residente do Serviço de Neurocirurgia do HSE. Aceite: 4-marco-1993.

Dr. José Carlos Brant Seggia - Av. Ary Parreiras 659 - 24230-321 Niterói RJ - Brasil. 
concepcionais, outros medicamentos e doenças próvias. Ao exame físico - Altura 1,67 m; peso $48 \mathrm{Kg}$; PA $100 \times 60 \mathrm{mmHg}$; pulso radial $80 \mathrm{bat} / \mathrm{min}$; frequência respiratória 16 inc/min. Eutrófica, normolínea, apresentando panículo adiposo e pêlos de distribuição normal; mancha congênita violácea na mama esquerda. Tórax: ritmo cardiaco regular em dois tempos, sem sopros; ausculta pulmonar sem alterações. Abdome: plano, indolor à palpação superficial e profunda, sem visceromegalias. Exame neurológico mostrando funções mentais superiores, marcha, estática, força, coordenaçăo, tono, reflexos e sensibilidade superficial e profunda normais. Sinais meningo-radiculares ausentes. Palpação de nervos periféricos sem alterações. Oftalmoscopia: mínimo apagamento da borda nasal da papila óptica bilateralmente, pulsos venosos ausentes que reapareciam pela compressão leve do globo ocular. Acuidade visual normal para perto e corrigida para longe por lentes. Demais nervos cranianos normais. Foi submetida a TC que revelou diminuição dos ventrículos laterais. Punção lombar mostrou LCR com pressão iniclal de $200 \mathrm{~mm}$ H2O: exame citológico e bioquímico normais.

Campimetria com aumento da mancha cega bilateralmente, mais evidente à esquerda (Fig. 1). O estudo de angiografia fluoresceínica foi considerado normal (Fig. 2). Os estudos hematológicos, sorológicos, hormonais e bioquímicos foram normais. Iniciada terapêtica com prednisona na dose $60 \mathrm{mg} /$ dia. No final do terceiro dia de tratamento apresentou intensa reação cutânea acompanhada de prurido intenso e aumento de peso obrigando à suspensấo da terapeutica. Substituida pelo glicerol a 50\%, por via oral na dose de $40 \mathrm{ml}$ quatro vezes ao
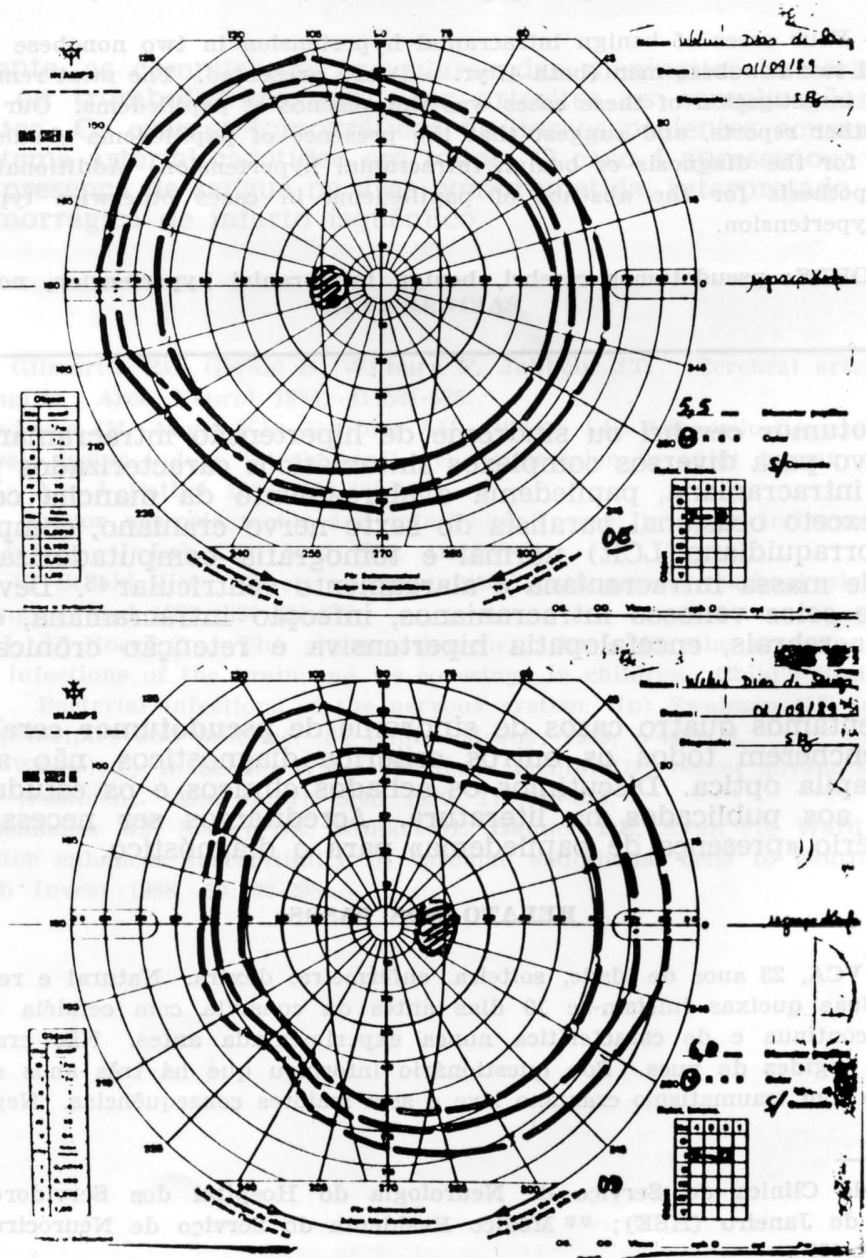

Fig. 1. Caso 1: campimetria visual. 

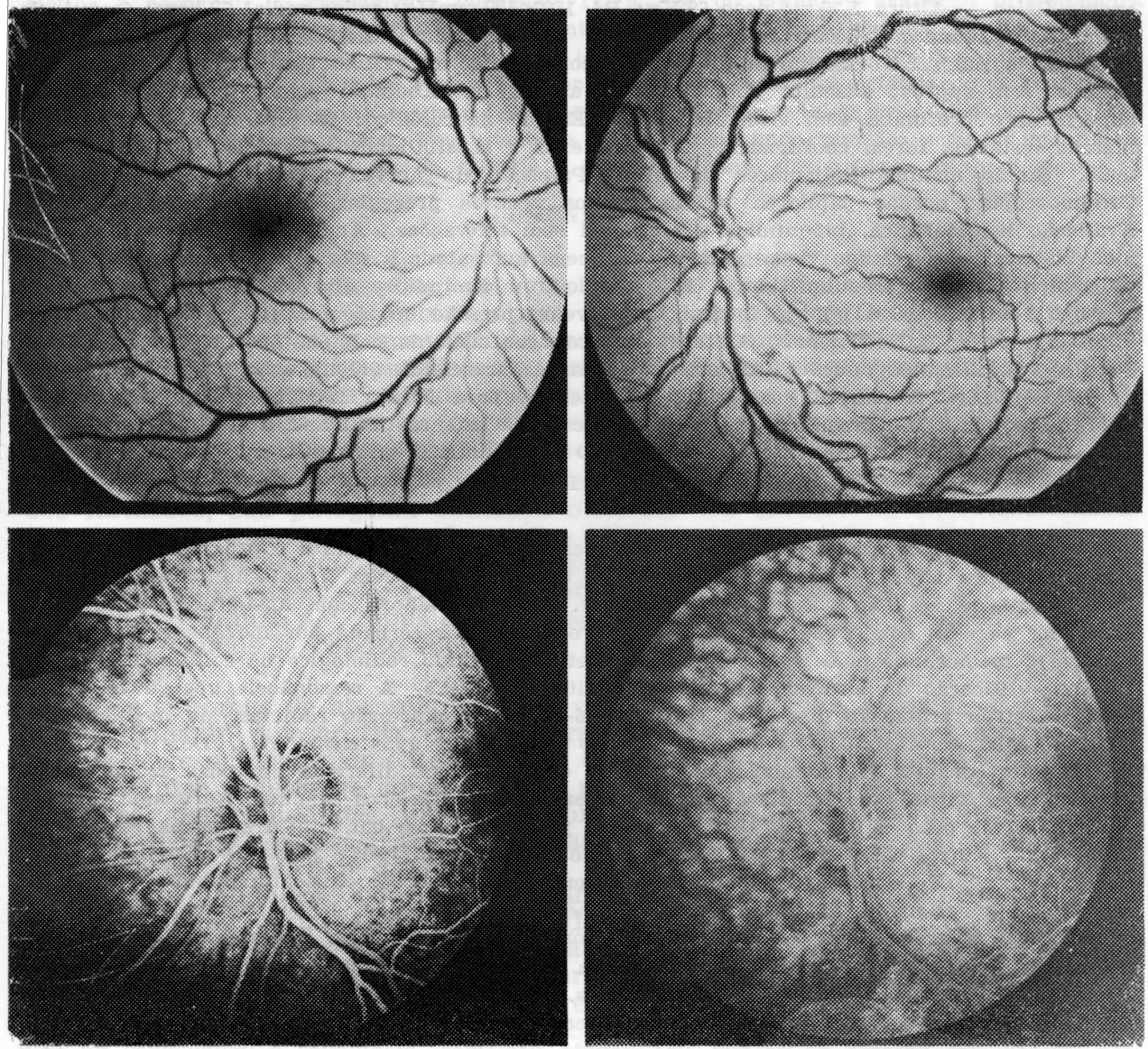

Fig. 2. Caso 1: fundoscopia; angiografia fluoresceínica.

dos oftalmológicos. $O$ tratamento foi irregular e interrompldo inadvertidamente quatro meses apos. Nova punção lombar revelou pressão do LCR de $230 \mathrm{~mm}$ H2O. Nesta ocasiăo observamos desaparecimento do pulso venoso à oftalmoscopia e aumento da mancha cega quando da realização do estudo do campo visual. Administramos acetozolamida na dose $750 \mathrm{mg} / \mathrm{dia}$, furosemida $40 \mathrm{mg} /$ dia e $\mathrm{KCl} 2 \mathrm{~g} /$ dia. Após um mês de tratamento houve melhora da cefaléia, reaparecimento do pulso venoso na oftalmoscopia, e queda da pressão do LCR (190 mm H2O). O acompanhamento fol realizado mensalmente e no final do quinto mês mostrava campimetria normal, pressão do LCR de $200 \mathrm{~mm}$ H2O e diminuição da cefaléla. Foi aumentada a dose de acetozolamida para $1000 \mathrm{mg} /$ dia o que levou a pressão intracraniana a $170 \mathrm{~mm} \mathbf{H 2 O}$ e desaparecimento da cefaléia. Dois anos após o início do quadro mantinha-se assintomática, apresentando exame neurológico, oftalmoscopia e campimetria normais. Suspensas as medicaçōes permaneceu assintomática por mais seis meses, sendo então submetida a novos exames de controle que revelaram TC do crânio e campimetria normais, LCR sem alteraçóes bioquimicas e pressóricas. Continua em acompanhamento periódico, sem modificação do perfil evolutivo.

Caso 2. ACA, dextro, branco, 47 anos de idade, solteiro, mecânico de automóveis, natural de Vitória. Suas queixas iniclaram-se 20 dias antes da consulta, com cefaleia holocraniana de tipo pulsátil, intensa, acompanhada de fosfenos e fotofobia, não responsiva a analgésicos comuns. Refere, há 6 anos, episódio semelhante cuja investigação teria demonstrado à TC «edema cerebrali. Teria sido medicado com dexametasona por duas semanas com desaparecimento dos sintomas. Novo estudo tomográfico do crânio, realizado um mês após, foi considerado «normal». portador de hipertensão arteríal de 4 anos de duração em uso irregular de hipotensores. Ao 
exame físico - Altura $1,70 \mathrm{~m}$; peso $69 \mathrm{Kg}$; PA $180 \times 120 \mathrm{mmHg}$; pulso radial 72 bpm; frequência respiratória $16 \mathrm{inc/min.} \mathrm{Eutrófico,} \mathrm{normolíneo,} \mathrm{apresentando} \mathrm{panículo} \mathrm{adiposo} \mathrm{e} \mathrm{pelos} \mathrm{de} \mathrm{dis-}$ tribuição normais. Normo-hidratado e normocorado. T6rax: ritmo cardiaco regular em dois tempos, sem sopros; ausculta pulmonar sem alterações. Abdome: plano, indolor à palpação superficial e profunda, sem visceromegalias. Exame neurológico: funções mentais superiores, marcha, estática, força, coordenaçăo, tono, reflexos e sensibilidade superficial e profunda normais. Sinais meningo-radiculares ausentes. Palpação de nervos periféricos sem alteraçð̄es. Oftalmoscopia: bordas papilares nitidas, pulsos venosos presentes, adelgacamento arteriolar. Avaliação hematológica, bioquímica e hormonal sem alteraçóes. Angiografia fuoresceínica normal. Alteraçōes no campo visual com mancha cega aumentada à esquerda e redução concêntrica do campo visual. Foi submetido a TC que mostrou diminuicăo dos ventrículos laterais $e$ do terceiro ventrículo e a punção lombar que revelou pressão de $250 \mathrm{~mm}$ H2O, estudos citológico e bioquímico do LCR normais. Obteve melhora acentuada da cefaléia após a punçăo lombar. Proposto esquema de punçóes lombares semanais e furosemida (40 mg/dia). Observamos queda da pressão para $170 \mathrm{~mm}$ H2O na segunda punçăo e para $90 \mathrm{~mm}$ H2O na quinta. Permaneceu por algum tempo assintomático, reaparecendo cefaléia intensa. Submetido a nova pungåo lombar com LCR laboratorialmente normal, pressão de $70 \mathrm{mmH2O}$. Suspensa a furosemida, melhora da cefaléia. Vinte dias após nova punção com pressăo de $80 \mathrm{~mm}$ H2O. Mantendo-se atualmente assintomático.

Caso 3. MLA, branca, 35 anos, dextra, casada, psicóloga, natural e procedente do Rio de Janeiro. Cinco anos antes da consulta notou cefaléia nucal com irradiação para o vértex e face lateral do cranio, descrita como capacete. Obteve melhora com ansiolíticos. Dez meses atrás dor cervical com irradiação para o ombro direito, exacerbada por movimentos da coluna cervical e melhorando pelo repouso. Foi submetida a estudo radiológico da coluna cervical que revelou retificaçăo da lordose cervical e osteófitos em C4-C5. A ressonância nuclear magnética (RM) mostrou protrusão discal em C5-C6 e C6-C7 sem compressão radicular ou medular. Foi colocado colar cervical. Houve desaparecimento das queixas após trinta dias. Dois meses antes da presente consulta foi notada cefaléia forte, descrita como diferente de todas as anteriores, holocraniana, surda, sem irradiação e não responsíva a analgésicos comuns. Ao exame físico Altura $1,65 \mathrm{~m}$; peso $45 \mathrm{Kg}$; PA $100 \times 70 \mathrm{mmHg}$; pulso radial $90 \mathrm{bat} / \mathrm{min}$; frequência respiratória $14 \mathrm{inc/min}$. Eutrófica, normolínea, apresentando panículo adiposo $\Theta$ pêlos de distribuiçăo normal. Tórax: ritmo cardíaco regular em dois tempos, sem sopros; ausculta pulmonar sem alteraçōes. Abdome: plano, indolor à palpação superficial e profunda, sem visceromegalias. Exame neurológico mostrando funçōes mentais superiores, marcha, estática, força, coordenação, tono, refiexos e sensibilidade superficial e profunda normais. Sinais meningo-radiculares ausentes. Palpaçăo de nervos periféricos sem alteracões. Oftalmoscopia, campos visuais e angiografia fluoresceínica sem alteraçóes. Demaís nervos cranianos normais. RM do crânio normal. Punção lombar com pressão de $200 \mathrm{~mm}$ H2O, estudo citológico, bioquímico e imunológico do LCR normal. Iniciada, por via oral, furosemida na dose de $80 \mathrm{mg} / \mathrm{dia}$. Como não obteve melhora da cefaléia, após 15 dias foi submetida a nova punção lombar que evidenciou pressão de $250 \mathrm{~mm}$ H2O. Acrescentamos à medicação acetazolamida por via oral na dose de $750 \mathrm{mg} / \mathrm{dia}$. Nova punção lombar realizada uma semana depois mostrou pressão de $100 \mathrm{~mm}$ H2O. Permanece, até a presente data, sem queixas com exame neurológico, campimetria e tela tangente normais.

Caso 4. LCS, branco, 47 anos, dextro, casado, engenheiro civil, natural e prccedente de Aracaju. Quarenta dias antes da consulta observou cefaléia frontal com irradiação para todo o cranio, definida como diferente de tcdas as anteriores e dando a sensação de pressão de dentro para fora, ocorrendo muitas vezes de madrugada e acordando-o. Tende a ser mais intensa de manhã, ao levantar-se, melhorando com a bipedestação e o correr do dia. Manifesta-se diariamente. Percebeu, em algumas ocasiōes, perturbaçōes visuais que descreve como «manchas escuras situadas difusamente nos campos da visão». Não melhora com analgésicos comuns e exacerba-se pelos esforços físicos. Sabe ser portador de hipertensão arterial há seis anos e submeteu-se a intervenção cirúrgica com implante de mamária após infarto aguđo do miocárdio em 1984. Ao exame físico - Altura $1,75 \mathrm{~m}$; peso $75 \mathrm{Kg}$; PA $130 \times 80 \mathrm{mmHg}$; pulso radial $80 \mathrm{bat} / \mathrm{min}$; frequência respiratória $16 \mathrm{inc} / \mathrm{min}$. Eutrófico, normolíneo, apresentando panículo adiposo e pêlos de distribuição normal. Tórax: ritmo cardíaco regular em dois tempos, sem sopros; ausculta pulmonar sem alterações. Abdome: plano, indolor à palpação superficial e profunda, sem visceromegalias. Exame neurológico revelando funções mentais superiores, marcha, estática, força, coordenação, tono, reflexos e sensibilidade superficial e profunda normais. Sinais meningo-radiculares ausentes. Palpação de nervos periféricos sem alterações. Oftalmoscopia: papila optica de bordas nitidas, vasos de aspecto e calibre normais, pulso venoso presente espontaneamente. Campos visuais sem alteraçóes. Demais nervos cranianos normais. Hemograma, velocidade de hemossedimentação, exames bioquímicos de rotina, urina, parasitológico de fezes, lipidograma, proteinograma, dosagem de hormônios tireoidiano, cortisol, pro- 
lactina e hormónio do crescimento normais. TC e RM do crânio e avaliação oftalmológica (pressão intra-ocular, campimetria, tela tangente e angiografia fluoresceínica) foram normais. Realizamos raquicentese que mostrou pressão inicial de $220 \mathrm{~mm}$ H2O a análises bioquímicas e imunológicas normais. Após a punção lombar ocorreu melhora da cefaléia, que reapareceu quatro dias depois. Foi enta realizada outra raquicentese que revelou pressão de $240 \mathrm{~mm} H 2 O$ e estudos laboratoriais normais. Propusemos e estamos realizando punções seriadas mantendo, até o momento, alívio acentuado da cefaléia e desaparecimento das queixas oftalmológicas.

\section{COMWNTARIOS}

Desde a descrição da síndrome de pseudotumor cerebral em 1897 por Quincke 7 muitos trabalhos têm sido publicados e, com o passar dos anos, aflorou um consenso quanto aos critérios para o seu diagnóstico. Os sinais e sintomas desta síndrome: cefaléia, aumento da pressão intracraniana, papiledema e alargamento de mancha cega, paralisia de sexto nervo craniano como única manifestação neurológica focal, LCR de composição normal e evidências radiológicas de ventrículos cerebrais normais ou diminuídos - são vistos, em mulheres jovens e obesas, em $90 \%$ dos casos. A ocorrência após a idade de 45 anos é rara.

Conquanto a sindrome esteja correlacionada a inúmeras doenças e a diversos fármacos, sua patogenia continua obscura. Na revisão da literatura encontramos, como causas, anemia ferropriva 8,32, uso de esteróides $9,15,51$ asma 12, gra. videz 16, menarca 17, disfunção menstrual 18,34, doença de Addison 26,53, uso de tetraciclina 30,39 , dissecação radical unilateral do pescoço 34 , obesidade 19,36, intoxicação pela vitamina $A^{38}$, lupus eritematoso sistêmico 5 , ao ácido nalidíxico 6 , uso de anticoncepcionais 55 , defeito metabólico congênito $52 \mathrm{e}$, a forma mais comum, a idiopática.

Os sintomas de apresentação são cefaléia e distúrbios visuais. A cefaléia é frequentemente pior ao despertar e agravada pela tosse e esforço, sendo usualmente suave ou eventualmente ausente. Contudo, em algumas séries 48 , a maioria dos pacientes a descreve como sendo a pior que já haviam experimentado e que podia ser distinguida das anteriores por sua pulsatilidade incomum, sua característica focal e pela associação a náuseas e rigidez do pescoço. Cerca de 5\% dos pacientes experimentam diminuição da acuidade visual associada ao edema de papila, esta diminuição pode ser permanente se persistir a hipertensão intracraniana. A perda visual pode ocorrer cedo ou tarde e a presença de hipertensão arterial é fator de risco significante para a perda visual. $\mathrm{Na}$ série de Corbett et al.10, cerca de $61 \%$ dos pacientes hipertensos com pseudotumor cerebri ficaram cegos. A observação de aumento da pressão intra-ocular pelo uso de corticosteróides sistêmicos pode ser fator de risco adicional 10.

O seguimento dos pacientes com pseudotumor cerebri foi inicialmente feito por medida da mancha cega, atribuída ao deslocamento dos elementos retinianos peripapilares pelo disco edemaciado 54 ou por desorganização retiniana 13 . $O$ alargamento persistente da mancha cega a despeito da resolução do papiledema fez desta mensuração instrumento não confiável para avaliar a terapêutica ou para predizer a pressão intracraniana. Na verdade, a mancha cega reflete mais o estado dos discos ópticos do que a pressão intracraniana; podemos citar ainda as alterações glióticas crônicas que mantêm o aumento de mancha cega, a despeito da resolução do papiledema e da normalização da pressão intracraniana 2,25.

Os sintomas visuais mais comuns são visão borrada que pode ser manifestação do papiledema e, mais raramente, diplopia secundária a paralisia do nervo abducente. Quando o paciente apresenta episódios breves de perda visual, usualmente precipitada por tosse ou esforço, devemos considerar prenúncio de grave risco de perda visual. Os defeitos do campo visual vistos nesta entidade são: alargamento da mancha cega, defeito nasal inferior, defeito arqueado, constriçōes concêntricas, escotomas centrais ou ainda paracentrais e, por vezes, constrição dos campos periféricos. Devemos ressaltar que estas alterações são observadas ao exame de perimetria automatizada $e$, na maioria dos casos, ausente nos testes por confrontação 47 .

São evidências da síndrome de hipertensão intracraniana: papiledema, pressão do LCR acima de $200 \mathrm{~mm}$ Hg. e ocasionalmente paralisia do abducente. Á TC do crânio pode revelar ventrículos diminuídos (em fenda) ou normais 22 . Quando encontramos ventrículos alargados devemos colocar em dúvida o diagnóstico. Segundo alguns a condição é ususalmente autolimitada e tem bom prognóstico. Contudo, há vários estudos revelando, em percentual variável, perda visual permanente 28,46 . 
A análise da síndrome observada em nossos pacientes preenche todos os critérios acima descritos exceto o edema de papila. Devemos ainda ressaltar que outro aspecto não usual é o biotipo dos pacientes, haja visto que todos são magros em desacordo com a maioria dos casos já descritos. Embora possamos considerar que a ausência de pulso venoso ao exame fundoscópico seja elemento importante no perfil evolutivo da formação do edema de papila, não estamos completamente autorizados a considerá-1o isoladamente. Acreditamos que seu reaparecimento quando da normalização da pressão intracraniana faz pensar que talvez, em alguns de nossos casos, a presteza diagnóstica não tenha proporcionado tempo hábil para o aparecimento do edema de papila.

Numa revisão bibliográfica encontramos o estudo de Lipton e Michelson 31, em 1971, como primeira descrição de pseudotumor cerebral sem edema de papila; foi sugerida, como explicação, uma anormalidade congênita da bainha do nervo optico impedindo a comunicação com o espaço sub-aracnóideo. Encontramos 21 outros casos publicados por Scanarini et al.42, Johnston et al.27, Spence et al.44, Marcelis e Silberstein 33 . Como explicação para o não aparecimento do edema de papila, a razão sugerida foi a elevação transitória ou intermitente da pressão intracraniana, em níveis abaixo daquela necessária para produzir o edema de papila 44. Acreditamos que o Caso 1 obedeça à mesma explicação dos casos de Spence et al.44, já que o diagnóstico foi levantado e o tratamento iniciado em curto espaço de tempo. Para corroborar a idéia de elevação intermitente ou transitória da pressão intracraniana, notamos alterações momentâneas na medida da mancha cega por ocasião de estudo seriado de campimetria e tela tangente que realizamos. embora devamos considerar as restrições à metodologia, como já citamos.

interessante observar que cerca de $1 / 3$ dos casos tem remissão espontânea depois da primeira punçăo lombar. A maioria dos casos pode ser manejada por punçōes lombares de repetição, inicialmente diárias, depois de três em três dias, semanalmente e ainda mensais, quando necessário. Devemos retirar volume suficiente de LCR para baixar sua pressão a $180 \mathrm{~mm} H 20$. A retirada de $30 \mathrm{ml}$ de LCR nesses pacientes faz em geral a pressão intracraniana voltar ao normal. Entretanto, a produção normal do LCR é de $3,5 \mathrm{ml} / \mathrm{min}$ e neste ritmo de produção a pressão intracraniana retorna ao nivel prévio ou próximo dela ao fim de 90 minutos, anulando ou reduzindo portanto o efeito benéfico. Como explicar então aqueles casos quando uma única punção produz melhoras duráveis? Seriam duas as explicaçōes mais plausíveis: saída continuada de LCR através de pertuito persistente, até que sua oclusão ocorra; correção de eventuais distorçōes de estruturas intracranianas após a punção e o seu reaparecimento depois de algum tempo 43 . O paciente do Caso 2 apresentou, durante a evolução, hipotensão do LCR que acreditamos obedeça a persistência de pertuito ocasionando uma fístula.

Devemos ressaltar que os sinais e sintomas observados na sindrome de hipotensão do LCR são os mesmos da hipertensão e, portanto, podem causar num paciente como este, confusão diagnóstica se essa possibilidade não for lembrada. importante notar que a hipotensão do LCR pode ser uma das complicações após o tratamento por punções lombares de repetição. A terapêutica proposta para correção de possível pertuito após punção lombar é a injeção de pequena quantidade de sangue do paciente na região lombar, procedimento este que provocaria irritação local e fibrose reacional, ocluindo o orifício.

Nos pacientes em que não se obtiver resposta a estas medidas terapêuticas podemos tentar prednisona (40-60 mg/dia) ou dexametasona $(6-12 \mathrm{mg} / \mathrm{dia})$ com as ressalvas que mencionamos anteriormente. A resposta a estas drogas ocorre após uma semana. Devemos ressaltar que a retirada desta medicação deve ser gradual com a finalidade de prevenir o efeito rebote de aumento da pressão intracraniana e reaparecimento do papiledema. O glicerol por via oral, na dose de $15-60 \mathrm{ml}$ três a quatro vezes ao dia ou acetozolamida $(250 \mathrm{mg}$ três vezes ao dia) são outros tratamentos médicos propostos. Quando ocorre perda visual progressiva a despeito da terapêutica medicamentosa têm sido propostos descompressão subtemporal 11, "shunting" lombo-peritoneal 4,24 e descompressão do nervo óptico 29.

Em nossa primeira paciente obtivemos boa resposta à terapêutica proposta e ela permanece assintomática. No segundo paciente acreditamos que os dois episódios relatados tenham sido semelhantes e que o segundo episódio represente recidiva da síndrome de hipertensão intracraniana vista seis anos antes. A terceira paciente, até a presente data, continua em tratamento ainda sem controle 
absoluto da pressão intracraniana e mantém-se sintomática. O quarto paciente obteve melhoras acentuadas com as punções lombares que continuamos fazendo.

Acreditamos que devemos rever o critério de obrigatoriedade do "papiledema» para o diagnóstico da síndrome de pseudotumor cerebri. A existência de número razoável de casos desta síndrome sem edema de papila, já descritos na literatura médica, reforça tal afirmativa. Obviamente um novo problema passa a ser a indicação de punção lombar em pacientes em que a cefaléia é o sintoma principal e que os outros dados do exame neurológico estejam normais. Talvez um estudo mais detalhado das características da cefaléia, a detecção de alterações ao exame do campo visual e da tela tangente possam colaborar para uma indicação mais precisa deste procedimento.

Gostaríamos de realçar que uma única medida da pressão do LCR não deve ser considerada como critério diagnóstico definitivo. Para alguns autores só a análise de curvas de pressão do LCR teria real valor. A avaliação custo $\times$ benefício favorece, a nosso ver, a realização da punção lombar.

\section{REEERENCIAS}

1. Ahlskog JE, O'Neill BP. Pseudotumor cerebri. Ann Intern Med 1982, 97:249-256.

2. Arbit E, Ruff RL. A blind spot tester. J Neurol Neurosurg Psychiatry 1981, 44:93-99.

3. Arbit E, Ruff RL. A blind spot tester. J Neurol Neurosurg Psychiatry 1981, 44:92-93.

4. Ark GDV, Kempe LG, Smith DR. Pseudotumor cerebri treated with lumbar-peritoneal shunt. JAMA 1971, 217:1832-1834.

5. Bettman JW, Daroff BB, Sanders MD, Hoyt WF. Papilledema and asyntomatic intracranial hypertension in systemic lupus erythematosus. Arch Ophthalm 1968, 80:189.

6. Boreus LD, Sundstrom B. Intracrianl hypertension during treatment with nalidixic acid. Br Med J 1967, 2:744.

7. Bulens C, DeVries WAJ, van Crevel $\mathbf{H}$. Benign intracranial hypertension: a retrospective and follow-up study. J Neurol Sci 1979, 40:147.

8. Capriles LF. Intracrainal hypertension and iron-deficiency anemia: report of four cases. Arch Neurol 1963, 9:147-153.

9. Cohn GA. Pseudotumor cerebri in children secondary to administration of adrenal steroids. J Neurosurg 1963, 20:784-786.

10. Corbett JJ, Savino PJ, Thompson HS, Kansu T, Schatz NJ, Orr LS, Hopson D. Visual loss in pseudotumor cerebri: follow-up of 57 patients from 5 to 41 years and profile of 14 patients with permanent severe visual loss. Arch Neurol 1982, 39:461-474.

11. Dandy WE. Intracranial pressure without brain tumor. Am Surg 1987, 106:492-513.

12. Dees SC, Mackay HWJr. Occurence of pseudotumor cerebri during treatment of children with asthma by adrenal steroids. Pediatrics 1959, 23:1143-1151.

13. Friesen $L$, Holm M. Visual field defects associated with choroidal folds. In Glaser $J$ (ed) : Neuro-ophthalmology. Ed 9. St Louis: Mosby, 1973, p 248-253.

14. Goley KM, Posner JB. Does pseudotumor cerebri cause the empty sella syndrome? Neurology 1975, 25:565-569.

15. Greer $M$. Benign intracranial hypertension: II. Following corticosteroid therapy. Neurology 1963, 13:439-441.

16. Greer $M$. Benign intracranial hypertension: III. Pregnancy. Neurology 1963, 13:670-672.

17. Greer M. Benign intracranial hypertension: IV Menarche. Neurology 1964, 14:569-573.

18. Greer M. Benign intracranial hypertension: V. Menstrual dysfunction. Neurology 1964 $14: 668-673$.

19. Greer M. Benign intracranial hypertension: VI. Obesity. Neurology 1965, 15:382-388.

20. Hayreh SS. Pathogenesis of oedema of the optic disc (papilloedema). Br J Opthtalm 1964, 48:522-543.

21. Hoyt WF, Pont ME. Pseudopapilledema: anomalous elevation of optic disk. Pitfalls in diagnosis and management. JAMA 1962, 181:191-196.

22. Huckman MS, Fox JS, Ramsey RG, Penn RD. Computed tomography in the diagnosis of pseudotumor cerebri. Radiology 1976, 119:593-597.

23. Huckman MS, Fox JS, Ramsey RG, Penn RD. Computed tomography in the diagnosis of pseudotumor cerebri. Radiology 1976, 119:593-597.

24. Jackson I, Snodgrass SR. Peritoneal shunt in the treatment of hydrocephalus and increased intracranial pressure. $J$ Neurosurg 1955, 12:216-222.

25. Jefferson A, Clark J. Treatment of benign intracranial hypertension by dehydrating agents with particular reference to the measurement of improvement. J Neurol Neurosurg Psychiatry 1976, 39:627-639. 
26. Jefferson A. A clinical correlation between encephalopathy and papilloedema in Addison's disease. J Neurol Neurosurg Psychiatry 1956, 19:2-27.

27. Johnston I, Hawke S, Halmagyi M, Teo C. The pseudotumor syndrome: disorders of cerebrospinal fluid circulation causing intracranial hypertension without ventriculomegaly. Arch Neurol 1991, 48:740-747.

28. Katz B, van Patten $P$, Rothrock JR, Katzman $R$. Optic nerve head drusen and pseudotumor cerebri. Arch Neurol 1968, 45:7.

29. Kilpatrick CJ. Gptic nerve decompression in benign intracranial hypertension. Clin Exp Neurol 1981, 18:161.

30. Koch-Weser J, Gilmore EB. Benign intracranial hypertension in an adult after tetracycline therapy. JAMA 1967, 200:345-347.

31. Lipton HL, Miche:son PE. Pseudotumor cerebri synärome without papilledema. JAMA 1972, $220: 1591-1592$.

32. Lubeck MJ. Papilledema caused by iron-deficiency anemia. Trans Am Acad Ophthalm 1959, 63:306-310.

33. Marcelis J, Silberstein SD. Idiopathic intracranial hypertension without papilltdema. Arci Neurol 15y1, 48:392-399.

34. Marr WG, Chambers RG. Pseudotumor cerebri syndrome following unilateral radical neck dissection. Am J Ophthalm 1961, 51:604-611.

35. McCullagh EP. Menstrual edema with intracranial hypertension (pseudotumor cerebri): report of a case. Cleveland Clin Quart 1941, 8:202-212.

36. Meyer JS, Gotham J, Tazaki Y, Gotoh F. Cardiorespiratory syndrome of extreme obesity with papilledema. Neurology 1961, 11:950-958.

37. Miller SJH, Sanders MD, Fytche TJ. Fluorescein fundus photography in the detection of early papilloedema and its differentiation from pseudo-papilloedema. Lancet 1965 , $2: 651-654$.

38. Morrice Jr G, Havener WH, Kapetansky F. Vitamin A a cause of pseudotumor cerebri. JAMA 1960, 173:1802-1805.

39. O'Doherty NJ. Acute benign intracranial hypertension in an infant receiving tetracycline. Develop Med Child Neurol 1965, 7:677-680.

40. Repka MX, Miller NR. Pseudotumor cerebri. Am J Ophthalm 1984, 98:741-746.

41. Rosemberg MA, Savino PJ, Glaser JS. A clinical analysis of pseudopapilledema: I. Population, laterality, acuity, refrative error, ophthalmoscopic characteristics and coincident disease. Arch Ophthaim 1979, 97:65-70.

42. Scanarini M, Mingrino S, Davella $D$, Della Corte V. Benign intracranial hypertension without papilledema: case report. Neurosurgery 1979, 5:376-377.

43. Sorensen PS, Krogsaa B, Gjerris F. Clinical course and prognosis of pseudotumor cerebri: a prospective study of 24 patients. Acta Neurol Scand 1988, $77: 164-172$.

44. Spence JD, Amacher AL, Willis NR. Benign intracranial hypertension without papilledema: role of 24-hour cerebrospinal fiuid pressure monitoring in diagnosis and management. Neurosurgery 1980, 7:326-336.

45. Sullivan HC. Fatal tonsillar herniation in pseudotumor cerebri. Neurology 1991, 41: 1142-1144.

46. Troost BT, Sufit RL, Grand G. Sudden monocular visual loss in pseudotumor cerebri. Arch Neurol 1979, 36:440-442.

47. Tulanne WM. Idiopathic intracranial hypertension: a prospective study of 50 patients. Brain 1991, 114:155-180.

48. Tullane WM. The headache profile of idiopathic intracranial hypertension. Cephalalgia 1990, 10:331-335.

49. Van der Ark GD, Kempe LG, Smith DR. Pseudotumor cerebri treated with lumbar-peritoneal shunt. JAMA 1971, 217:1832-1834.

50. Vassilouthis J, Uttley D. Benign intracranial hypertension: clinical features and diagnosis using computed tomopraphy and treatment. Surg Neurol 1979, 12:389.

51. Walker AE, Adamkiewicz JJ. Pseudotumor cerebri associated with prolonged corticosteroid therapy. JAMA 1964, 188:779-784.

52. Walsh FB, Hoyt WF. Clinical neuro-ophthalmology. Ed 3. Baltimore: William and Wilkins, 1974, Vol 3, p 1938-1939.

53. Walsh FB. Papilledema associated with increased intracranial pressure in Addison's disease. Arch Ophthalm 1952, 47:86.

54. Wash FR, Hoyt $M$. Clinical neuro-ophthalmology. Baltimore: William and Wilkins, 1969, Vol 1, p 65 .

55. Weisberg LA. Benign intracranial hypertension. Medicine 1975, 54:197-207. 